Appl. Phys. 6, 297-304 (1975)

(C) by Springer-Verlag 1975

\title{
Gain Induced Stability of Active Plane-Parallel Resonators
}

\author{
W. J. Witteman and G. J. Ernst \\ Twente University of Technology Enschede, The Netherlands \\ Received 7 May 1974/Accepted 7 January 1975
}

\begin{abstract}
It has been observed for a plane-parallel resonator with a saturated medium that under certain conditions confined beams mainly concentrated near the axis are present. The experiments have been done with a sealed-off plane-parallel $\mathrm{CO}_{2}$ laser of one meter length and with an internal diameter of $20 \mathrm{~mm}$. Without gain variations such a system is unstable because of thermal defocussing by the heated gas. But due to sufficiently high gain variations the beam converges nevertheless. Since the gain variation increases with decreasing reflectivity confined beams near the optical axis are only observed at sufficiently low reflectivity. The experiments were done with a totally reflecting mirror and an outcoupling mirror having a reflectivity of, respectively, $90 \%, 80 \%, 50 \%$, and $36 \%$. For the high reflectivities the outcoupled beam is mainly concentrated near the edge with irregular density distributions. For the low reflectivity the beam is more or less radially symmetric, concentrated near the axis having decreasing intensity with the distance from the axis.

The behaviour can be understood by an analysis in which the constant and quadratic term of the complex propagation constant near the optical axis due to gain variations, dispersion and heat effects are taken into account.
\end{abstract}

The output beam of most conventional lasers having at least one curved mirror, and a nearly homogeneous active medium can be simply described in terms of Laguerre-Gaussian functions [1]. The Gaussian structure is sometimes also observed for systems having non-uniform gain or refractive index. In general, these are media with axial symmetry.

The complex propagation constant of such a medium describing the relevant properties can be expanded into even powers of the radial distance from the axis. If the variations are sufficiently slow in the vicinity of the beams or if the beam is mainly concentrated near the axis like the Gaussian mode we may only keep the constant and quadratic terms. Satisfying this condition the Gaussian structure of a mode is preserved even though its spot size, wave front curvature, amplitude and phase are significantly altered by the axially symmetric inhomogeneous active medium. As we described previously [2] a Gaussian mode with a finite beam size can be obtained for any quadratic gain profile and any set of curved and/or flat mirrors. In the present work we are mainly interested in the influence of medium parameters on the beam profile of an active plane-parallel resonator. In the case of a defocussing index profile due to thermal effects, as may occur in $\mathrm{cw}$ solid state and gas lasers, the planeparallel system is, neglecting gain variations, unstable. However, it will be shown that in the presence of a gain profile confined beams concentrated near the axis can be found. This demonstrates the importance of gain on the output of a laser system. Experimental studies [3] with a plane-parallel xenon laser having a profile with the highest gain at the centre (positive profile) have already shown that a confined beam is possible. It is, however, also possible to obtain stable oscillations in a plane-parallel resonator with a medium having a profile with the lowest gain at the centre (negative profile) [2]. Although one might expect at first glance that such a medium would tend to broaden the beam diameter indefinitely, it turns out that for 
such a medium the wave front converges and, hence, a confined beam is obtained nevertheless. This can be observed with a plane-parallel resonator and a saturated medium. Saturation of the medium by the lowestorder mode causes the gain to increase with the distance from the axis. Apparently, this gain profile converges the wave front and hence a confined beam is obtained. The experiments show that these confined beams can only be observed as long as the reflectivity of the outcoupling mirror is sufficiently low.

The problem of finding the field distribution in a planeparallel resonator with finite apertures and a gain saturated medium has been treated numerically by Fox and Li [4]. They found for such a system that starting from the Kirchhoff-Fresnel diffraction fromula stable oscillations with a field distribution descreasing with the distance from the central axis are possible. Although, as we mentioned already, in a plane-parallel resonator a Gaussian mode with finite beam size can be obtained for any gain profile there has been no discussion on the stability of the modes, i.e. how does an oscillation start and how does it react on deviations from the steady state. When one looks at the behaviour of Gaussian modes in an infinitely long amplifying medium there is a large distinction between the two opposite gain profiles, because for a positive gain profile any Gaussian beam will fluctuate more or less around the stationary value, but will finally settle down to equilibrium [5], whereas for a negative profile any arbitrary Gaussian profile can rapidly lead to deterioration of the beam mode [6], unless the initial beam parameters are just equal to a steady state solution with finite beam size. However, such a description of the behaviour of Gaussian modes is not adequate in describing stability of modes in a practical planeparallel laser oscillator.

The situation here is very different because the system itself builds up the oscillations and the diffraction losses of finite apertures will certainly play an important role in the competition phenomena of an oscillating field settling down to equilibrium. In the final steady state these diffraction losses may be minimum. Thus, although for a steady state the mirror aperture can be large, as compared with the beam size, so that its influence on the beam parameters is very small, its finiteness may never be neglected in considering the stability of the steady state.

For the observed phenomena we will not discuss quantitatively the stability of the steady state but only analyse the conditions for which a steady state may be possible. This is done by an analysis in which the constant and quadratic terms of the complex propagation constant near the optical axis due to gain variations, dispersion and heat effects are taken into account. In this approximation it is found that, in spite of the thermal defocussing of the medium, confined beams can be obtained as long as these thermal effects are below a certain limit given by the gain variation or outcoupling reflectivity. Further it is found that due to the convergence of the wave front a considerable amount of radial radiation transport takes place [7]. For large intensities this transport at the optical axis is equal to the radiation gained directly from the medium, independent of thermal lensing.

With the used approximations the analysis is rather simple and contributes, at least qualitatively, to the understanding of the physics involved.

\section{Derivation of Medium Parameters}

In the following we assume that the beam is paraxially propagating more or less parallel to the $z$-axis. The properties of the active medium are axially symmetric. We are interested in finding a field distribution satisfying the wave equation that has the largest field strength near the axis and a decreasing field with the distance from the axis. The complex propagation constant which has a large bearing on the beam parameters is expanded into even powers of the distance $r$ from the axis. Near the optical axis, where the field is strongest, the constant and quadratic terms are most important. It is assumed that in first approximation to describe the field distribution near the axis these two terms are most important and that higher-order terms can be neglected. This approximation will certainly hold for large Fresnel numbers where the interference of off-axial rays is small. Applying these assumptions a solution of the wave equation satisfying the boundary conditions will be a Gaussian field distribution [2]. Although the experimental observations indicate that only close to the axis the intensity distribution is more or less Gaussian we start from this approach in order to get information on the gain induced stability conditions of a plane-parallel resonator.

The strong influence of cross relaxation on the gain saturation allows efficient molecular systems with a Doppler profile to be treated as homogeneously broadened [8]. The amplitude gain as a function of the radial distance $r$ from the axis is given by

$\alpha=\frac{\frac{1}{2} g G}{1+g I / I_{\mathrm{S}}}$, 
where $G, g, I$, and $I_{S}$ are, respectively, the smallsignal intensity gain, the line shape function, the radiation intensity and the saturation parameter. The gain will be expressed in a power series of radial distance $r$ from the axis. Since all processes contributing to the gain are axially symmetric the power series contains only even powers of $r$. Keeping again only the constant and quadratic terms we substitute

$G=G_{0}\left(1+\frac{1}{2} \gamma r^{2}\right)$

and

$I_{S}=I_{0}\left(1+\frac{1}{2} \delta r^{2}\right)$

into (1). Taking for the beam a Gaussian distribution like $I=I_{C} \exp \left(-2 r^{2} / w^{2}\right)$ we obtain for the constant term

$\alpha_{0}=\frac{\frac{1}{2} g G_{0}}{1+g I^{\prime}}$

and for the quadratic term:

$\alpha_{2}=\frac{\frac{1}{2} g G_{0}}{1+g I^{\prime}}\left\{\gamma+\frac{1}{1+g I^{\prime}}\left(\delta+\frac{4}{w^{2}}\right) g I^{\prime}\right\}$,

where $I^{\prime}=I_{\mathrm{C}} / I_{0}$.

In most practical cases having large discharge currents and a tube diameter larger than $w$ one may neglect the effect of $\gamma$ and $\delta$ on $\alpha_{2}$ as compared with that of $4 / w^{2}$, so we use the relation

$\alpha_{2}=\frac{2 g^{2} G_{0} I^{\prime}}{w^{2}\left(1+g I^{\prime}\right)^{2}}$.

As a consequence of this gain profile we also have to take into account the associated quadratic term of the dispersion of the medium. The contribution of this part of the dispersion to the index profile depends on the line shape and on the difference of the oscillating frequency and the central frequency. It turns out that the quadratic term of the index profile as caused by the gain profile is proportional to $\alpha_{2}$ and may be given by [7]

$n_{2}=\frac{\lambda \alpha_{2}}{2 \pi} F(\eta)$,

where $F$ is dimensionless, describing the line shape of the medium. In the case of a Doppler-broadened line we find

$F(\eta)=\frac{1}{\sqrt{\pi}} \int_{0}^{n} e^{x^{2}} d x$ where $\eta=2 \sqrt{\ln 2}\left(v-v_{0}\right) / \Delta v_{D} ; v$ is the frequency, $v_{0}$ is the central frequency and $\Delta v_{D}$ is the Doppler line width.

For the following derivations it turns out to be advantageous to use dimensionless parameters for the beam width as well as for the gain and index parameters [2]. We use for Gaussian beams

$Q=\frac{\lambda L}{\pi w^{2}}$

and

$U=\frac{L}{R_{c}}$,

where $L$ is the distance between the mirrors, and $R_{c}$ is the radius of curvature of the wave front.

The dimensionless gain parameter is defined as

$\alpha=\frac{\lambda \alpha_{2}}{2 \pi} L^{2}$.

Substituting (5) and (7) into (3) and using (2) we find

$\alpha=\frac{2 L \alpha_{0} g I^{\prime} Q}{1+g I^{\prime}}$.

Similarly, we write the dispersive part of the refractive index given by (4) in dimensionless form as

$\beta_{d}=\alpha F(\eta)$,

where

$\beta_{d}=n_{2} L^{2}$

The quadratic term of the refractive index of the medium $n_{t}$, which may be due to thermal heating, can be written in dimensionless form as

$\beta_{t}=n_{t} L^{2}$.

The total quadratic term of the refractive index profile is given by the sum of (9) and (10)

$\beta=\beta_{t}+\alpha F(\eta)$.

\section{The Beam Equations of a Propagating Gaussian Beam}

Using Kogelnik's ABCD-law in dimensionless form for the propagation of a Gaussian beam through a lenslike laser medium with the complex beam parameter defined as

$$
P=U-i Q
$$


we find

$P_{2}=\frac{C+D P_{1}}{A+B P_{1}}$,

where $P_{1}$ and $P_{2}$ are, respectively, the "input" and "output" beam parameters and where the coefficients are the elements of the paraxial ray matrix [2].

In the case of a plane-parallel resonator we have for the steady state the condition that $P$ is constant along the propagation axis. For the medium of length $L$ the matrix in dimensionless form can be written as

$\left(\begin{array}{ll}A & B \\ C & D\end{array}\right)$

$=\left(\begin{array}{lc}\cos (-\beta-i \alpha)^{\frac{1}{2}} & (-\beta-i \alpha)^{-\frac{1}{2}} \sin (-\beta-i \alpha)^{\frac{1}{2}} \\ -(-\beta-i \alpha)^{\frac{1}{2}} \sin (-\beta-i \alpha)^{\frac{1}{2}} & \cos (-\beta-i \alpha)^{\frac{1}{2}}\end{array}\right)$

Substituting (14) into (13) and applying the condition that $P$ is constant, we find

$P= \pm(\beta+i \alpha)^{\frac{1}{2}}$.

The choice of the solution for (15) depends on the sign of the imaginary part. Since $\alpha$ in our experiments is always positive (i.e. gain increases with the distance from the axis) only the solution of $P$ with the minus sign has a negative imaginary part and thus a positive beam waist. We find

$U=-\left(\alpha^{2}+\beta^{2}\right)^{\frac{1}{4}} \cos \phi$,

$Q=\left(\alpha^{2}+\beta^{2}\right)^{\frac{1}{4}} \sin \phi$,

where

$\phi=\frac{1}{2} \tan ^{-1}(\alpha / \beta)$.

In a previous publication [7] we have shown that the incremental gain can be described by the equation

$\frac{1}{I} \frac{d I}{d \xi}=2\left(\alpha_{0} L-U\right)$,

where

$\xi=\frac{z}{L}$.

In a laser system the standing wave consists of a running wave in the $+\xi$-direction and one in the $-\xi$-direction. If we write down the incremental gain for both running waves we obtain

$\left(\frac{1}{I} \frac{d I}{d \xi}\right)_{+}=-\left(\frac{1}{I} \frac{d I}{d \xi}\right)_{-}$

because the gain is isotropic and $U$ is constant.
From (20) we find that the product of the intensities of the two running waves is constant along the axis,

$I_{+} \cdot I_{-}=$constant

In the following we consider a system having one totally reflecting mirror and one outcoupling mirror with reflectivity $R$. For such a system the maximum relative variation of the sum of the two intensities is equal to $\frac{1}{2} \sqrt{R}(1+1 / R)$. From this it is seen that the larger the reflectivity the smaller the maximum relative variation of this sum. Even for $R=50 \%$ the sum of the two intensities is constant within $5 \%$. Therefore in the following we shall approximate the sum of the two intensities as constant. This approximation simplifies the analysis considerably.

Integrating (19) over a round trip through the resonator, we obtain

$4 \alpha_{0} L=4 U-\ln R$.

The left-hand term of (21) represents the radiation gained directly from the medium. The first term on the right hand side describes the radial radiation transport, whereas the last term represents the outcoupling loss. In laser systems having no gain profile at any place between the mirrors, the value of the radius of curvature of the wave front of a running wave to the left is equal to minus the value of the wave running to the right, i.e. the two wave fronts coincide. Consequently, the integral becomes zero and there is no radial radiation transport. However, for lasers having a gain profile this integral represents the net radial radiation transport, which-as we shall see--is comparable to, and for large small-signal gain, equal to the radiation gained directly from the medium.

Substituting (16) into (21) and using (11) we obtain $\left[\alpha^{2}+\left(\alpha F+\beta_{t}\right)^{2}\right]^{\frac{1}{4}} \cos \phi=-\frac{1}{4} \ln R-\alpha_{0} L$.

Substituting (17) into (8) we find

$\left[\alpha^{2}+\left(\alpha F+\beta_{t}\right)^{2}\right]^{\frac{1}{4}} \sin \phi=\frac{\alpha}{2 L \alpha_{0}} \frac{1+g I^{\prime}}{g I^{\prime}}$.

\section{Solution for the Beam Parameters}

The Eqs. (22), (23), and (2) describe, in principle, the intensity characteristics of the laser system as well as the corresponding beam width. Let us first calculate the intensity characteristics. Multiplying (22) by (23) we get

$\frac{1}{2}\left[1+\left(\frac{\alpha F+\beta_{t}}{\alpha}\right)^{2}\right]^{\frac{1}{2}} \sin 2 \phi=\frac{1+g I^{\prime}}{2 L \alpha_{0} g I^{\prime}}\left(\frac{1}{4} \ln R+\alpha_{0} L\right)$. 
Substituting (18) for $\phi$ we find that the left-hand side of (24) equals to $\frac{1}{2}$. The derivation of the intensity characteristics is now straightforward. Substituting (2) for $\alpha_{0}$ we obtain finally for the radiation intensity at the optical axis

$I^{\prime}=-g^{-1}-\frac{2 G_{0} L}{\ln R}\left[1+\left(1+\frac{\ln R}{2 G_{0} L} g^{-1}\right)^{\frac{1}{2}}\right]$.

It is seen that the intensity is independent of thermal effects and dispersion, as given by F. Further, if the line shape function $g$ is symmetric, having a maximum for the central frequency $(\eta=0)$, the intensity $I^{\prime}$ is also a symmetric function of $\eta$, having a maximum for $\eta=0$. The characteristics given by (25) are plotted in Fig. 1 for various values of the gain loss parameter $G_{0} L / \ln R$ as a function of the frequency shift. The plots have been made for a Doppler-broadened line.

We now look for an explicit expression for the beam parameter $Q$. For this purpose we first solve $\alpha$ from (22) and (23). Substituting (18) into (23), we find

$\alpha=-\frac{F}{2 Q^{2}}+\left(\frac{F^{2}}{4 \varrho^{4}}+\frac{1}{4 \varrho^{4}}-\frac{\beta_{t}}{\varrho^{2}}\right)^{\frac{1}{2}}$,

where

$\varrho=\frac{\left(1+g I^{\prime}\right)^{2}}{L g^{2} G_{0} I^{\prime}}$.

Next we substitute (26) into (23) and find for $Q$

$Q=-\frac{F}{2 \varrho}+\left(\frac{F^{2}}{4 \varrho^{2}}+\frac{1}{4 \varrho^{2}}-\beta_{t}\right)^{\frac{1}{2}}$.

From this we conclude that a finite beam width is always found if $\beta$ is negative. In the case of a defocussing medium, $\beta>0$, we find a finite beam if

$\beta_{t}<\frac{\left(L g^{2} G_{0} I^{\prime}\right)^{2}}{4\left(1+g I^{\prime}\right)^{4}}$

where $I^{\prime}$ is given by (25).

The beam parameter $Q$ is plotted in Fig. 2 as a function of the reflectivity $R$ for various values of the smallsignal gain $G_{0} L$. The plots are made for $\beta_{t}=0$ and for maximum radiation, i.e. at the central frequency, where $\eta=0, g=1$ and $F=0$. Because of the constant intensity approximation the theory is invalid at low reflectivity. The lower limit of $R=0.3$ in the figure is somewhat arbitrary. Further, the laser output can be obtained by calculating the transmitted part of the intensity and integrating it over the cross section of the

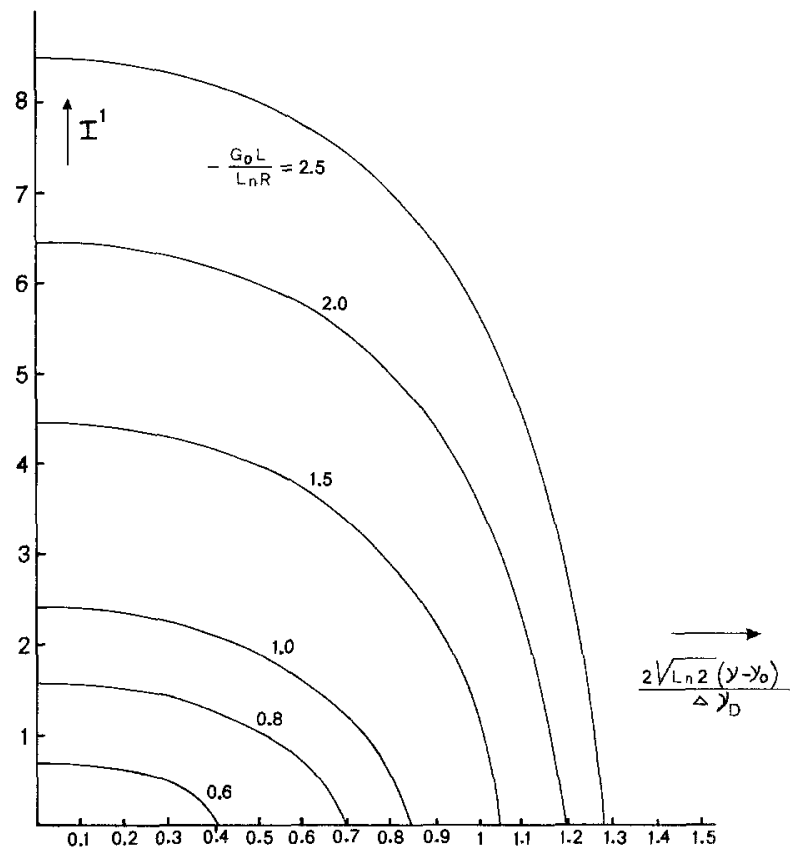

Fig. 1. The output characteristics of a plane-parallel resonator with the ratio of the small-signal gain and the outcoupling $\left(-G_{0} L / \ln R\right)$ as parameter. The characteristics are plotted as a function of the normalized frequency of a Doppler-broadened gain profile. The results are independent of thermal defocussing

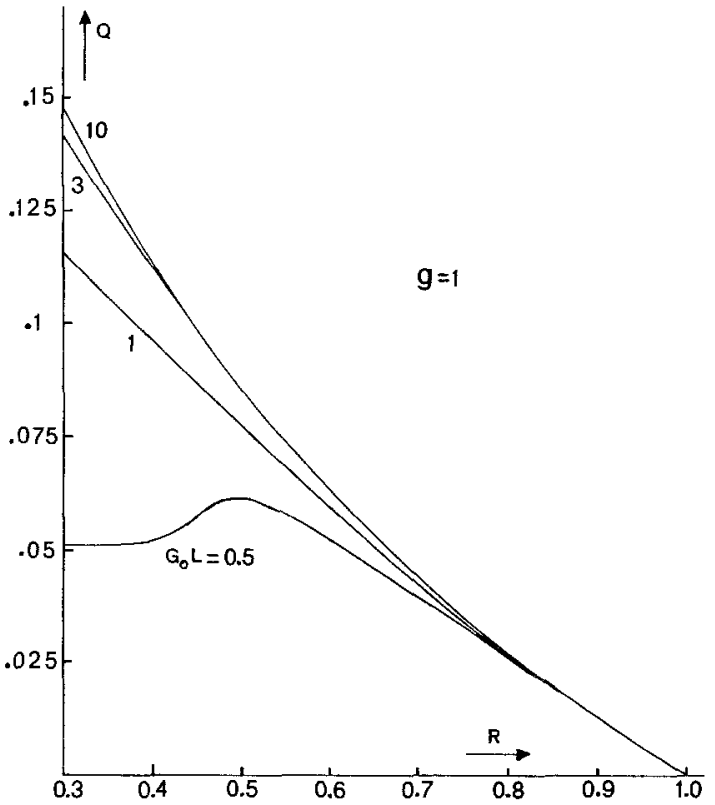

Fig. 2. The beam parameter $Q=\lambda L / \pi w^{2}$ as a function of reflectivity with the small-signal gain $G_{0} L$, i.e. the intensity gain over the length of the tube as parameter. The $Q$ 's are plotted for the central frequency and in the absence of defocussing $\left(\beta_{t}=0\right)$ 


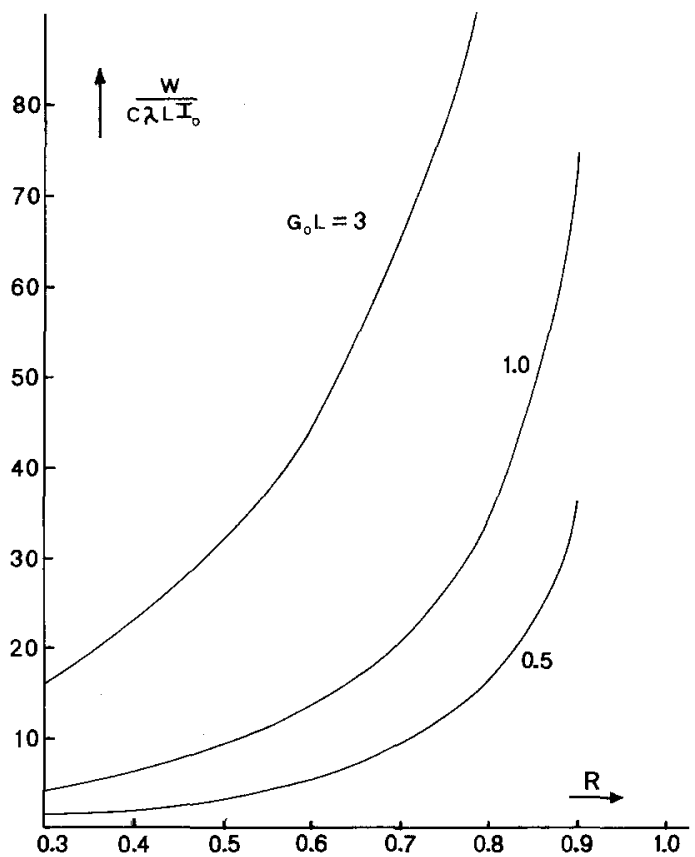

Fig. 3. The dimensionless output power is plotted against $R$ for various values of the small-signal gain $G_{0} L$. The total output is given by $W$ and the saturation parameter is given by $I_{0}$. The beam diameter increases strongly with $R$ because of decreasing gain. Hence the fast increase of $W$ with $R$

beam. Since $I^{\prime}$ is the sum of the two running waves, we find the outcoupling intensity $I_{\text {out }}$ given by

$\frac{I_{\mathrm{out}}}{I_{0}}=\frac{1-R}{1+R} I^{\prime}$.

Integrating over the cross section and using (5) we find for the output power $W$

$\frac{W}{c \lambda L I_{0}}=\frac{(1-R) Q I^{\prime}}{2(1+R)}$.

The dimensionless output is plotted in Fig. 3 for maximum intensity $I^{\prime}(\eta=0)$ and in the absence of an index profile $\left(\beta_{t}=0\right)$. The reason that the output power $W$ increases so strongly with $R$ is due to the fact that a decreasing gain profile results from an increasing reflectivity, which on its turn leads to a wider beam.

\section{Beam Parameter for High Gain Systems}

For many cases of practical interest where $-G_{0} L / \ln R \gg g^{-1}$ the above derivations of laser parameters simplify considerably. Equation (25) becomes now

$$
I^{\prime}=-\frac{4 G_{0} L}{\ln R} .
$$

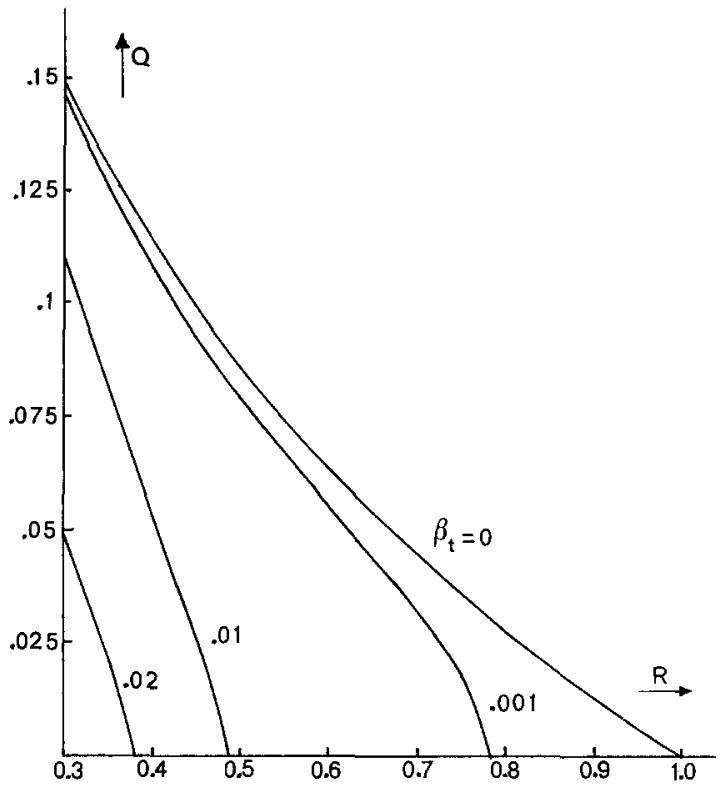

Fig. 4. The beam parameter $Q$ is plotted for highly saturated systems as a function of $R$ with the defocussing term $\beta_{t}$ as parameter

It is interesting to note that, if the radial radiation transport was not taken into account, the intensity at the optical axis would by given by

$I^{\prime \prime}=-\frac{2 G_{0} L}{\ln R}$.

Further, it is seen from (22) and (23) that the radial radiation transport is equal to the radiation gained directly from the medium. From (22) we find also that

$U=\frac{\ln R}{8}$.

The condition for finding a finite beam size reduces to the simple expression

$\beta_{t}<\left(\frac{\ln R}{8}\right)^{2}$.

Thus for high gain systems the radius of curvature of the beam and the maximum allowable value of the defocussing parameter are only a function of the reflectivity.

Finally the beam parameter $Q$ becomes at the central frequency $(\eta=0)$

$Q=\left[\left(\frac{\ln R}{8}\right)^{2}-\beta_{t}\right\}^{\frac{1}{2}}$.

These values of $Q$ are plotted in Fig. 4 for different values of $\beta_{t}$ as a function of $R$. We note a large influence of $\beta_{t}$ on $Q$. 


\section{Experimental Results}

In the preceding sections we have given a feasability study for obtaining gain induced confined beams in plane-parallel resonators. Attempting to realize this experimentally we choose a $\mathrm{CO}_{2}$ active medium because of its high gain, saturation properties and its practically homogeneously broadened profile.

The analysis shows that in order to get a narrow beam of limited cross section that fits a normal discharge tube the gain profile must be sufficiently large. This requires low outcoupling reflectivity. On the other hand, saturation of the medium requires high reflectivity. Increasing the length of the laser cavity, so that low reflection together with high intensity and therefore a saturated medium is obtained, leads also to a wider beam. From the definition of $Q$, which can be considered as an inverse Fresnel number of the beam, we see that the beam width is proportional to the square root of the cavity length. Compromising these effects of the beam parameters we find, as a reasonable choice, a discharge tube of one meter length and an internal cross section of $20 \mathrm{~mm}$ provided with internal mirrors. The reflectivity of outcoupling mirror should be less than $60 \%$. As an active medium we choose 1.5 torr $\mathrm{CO}_{2}, 2$ torr $\mathrm{N}_{2}$, and 12 torr $\mathrm{He}$.

This system with internal plane-parallel mirrors was investigated for four different relectivities of the outcoupling mirror, i.e. $36 \%, 50 \%, 80 \%$, and $90 \%$. From the experimental study we learned that confined beams are not easily obtained. With a normal cw discharge the output beam is mainly concentrated near the edge with irregular density distributions, which are difficult to define, even at low excitation rate. There was no excitation condition for which a confined stable beam could be observed. The observations were more or less similar for all reflectivities except for the lowest one. With the outcoupling reflectivity of $36 \%$ the system did not lase at all. Apparently the losses were too high. We concluded that the defocussing effect of the heated gas prevented a stable oscillation pattern if possible at all. Estimating the quadratic term of the refractive index due to thermal heating we find $\beta_{t}$ in between 0.005 and 0.01 depending on discharge conditions. According to Fig. 4 these values are too large to obtain a converging beam. We then used a square-wave discharge current with an excitation current of $20 \mathrm{~mA}$ during a time of 1 millisecond followed by a low discharge current of $2 \mathrm{~mA}$ during 7 milliseconds. The time constant for building up a stationary index profile is much longer than one millisecond, whereas the "dead time" of 7 milliseconds reduces the defocussing parameter $\beta_{t}$ considerably. The small current of $2 \mathrm{~mA}$ in between the excitation periods was found necessary to control the discharge. Otherwise the starting current, which first has to ionize part of the molecules in order to get a conducting medium, is not axially symmetric but irregular through the tube. The excitation and relevant energy processes are much faster than one millisecond, so that during the one millisecond a quasi continuous excitation process is obtained. Using this squarewave discharge current we observed indeed confined beams near the axis for the two low reflectivities of $36 \%$ and $50 \%$. Higher reflectivities still show a pattern comparable with the previous experiments. In Fig. 5 we show some measurements done for the above conditions. We measured the output beam for three

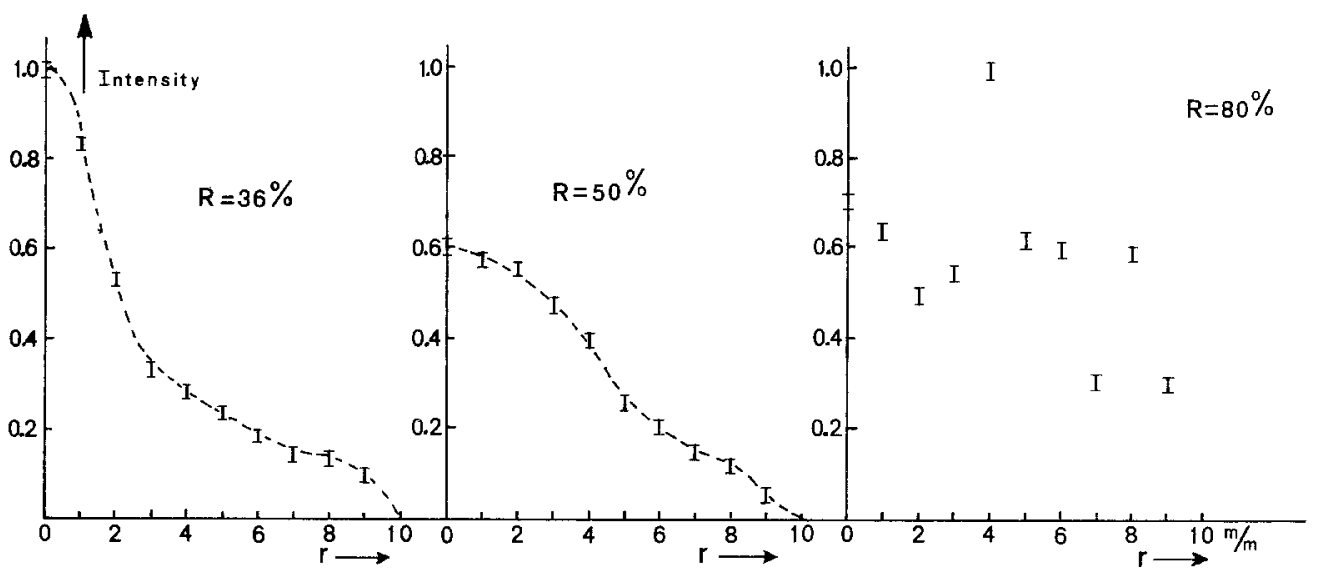

Fig. 5. The measured intensities of the output beam of a plane-parallel resonator having one totally reflecting mirror and an outcoupling mirror with reflectivity of respectively, $36 \%, 50 \%$, and $80 \%$. The intensities in arbitrary units are measured per unit area of concentric rings of about $0.5 \mathrm{~mm}$ thickness 


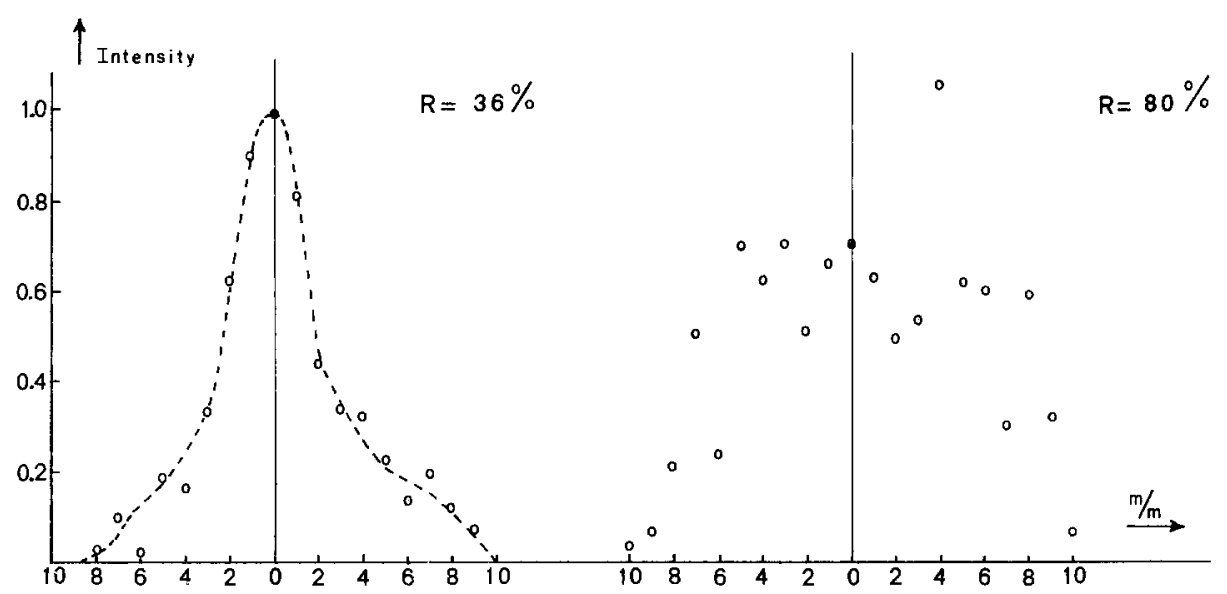

Fig. 6. The measured intensity across the output beam in arbitrary units for two outcoupling reflectivities of $36 \%$ and $80 \%$, respectively. The intensities are measured by means of a small hole of about $0.2 \mathrm{~mm}$ diameter

different reflectivities, i.e. $36 \%, 50 \%$, and $80 \%$. The intensities as a function of the radius are measured per unit area of concentric rings of about $0.5 \mathrm{~mm}$ thickness. It is seen that a reflectivity of $36 \%$ gives a narrower beam than a $50 \%$ reflectivity, which is in agreement with the above analysis. The radiation intensities as a function of the radial distance were also obtained by measuring the radiation coming through a hole of about $0.2 \mathrm{~mm}$ diameter. The results we obtained by scanning across the beams are plotted in Fig. 6. Also visualizing the beams on luminous material shows maximum intensity at the centre only for low reflectivities. Evaluating an effective beam width from the measurements by comparing the central part of the measurements with a Gaussian beam we find $w_{M} \simeq 8.5 \mathrm{~mm}$ for $50 \%$ and $w_{M} \simeq 4.5 \mathrm{~mm}$ for $36 \%$ reflectivity. The output powers were 18 Watts for $36 \%$ and 23 Watts for $50 \%$ reflectivity. The efficiences were $9 \%$ and $12 \%$, respectivity. These output and efficiences are relatively high for systems that are highly overcoupled.

\section{Discussion}

The experimental results are in substantial agreement with the theoretical predictions. The influence of medium and cavity parameters on the conditions to obtain confined beams has been, within certain limits, studied theoretically and experimentally. The theoretical results that the thermal effects have to be below a certain limit, given by the gain profile or outcoupling reflectivity, and further, that the beam width increases with increasing reflectivity are also found experimentally. Quantitatively good agreement between the experiment with $R=50 \%$ and Fig. 2 is found, if we estimate, under the applied discharge conditions ,the value for $G_{0} L$ of about 1 . For the smaller reflectivity of $36 \%$ the deviation of the beam width is about $25 \%$, which is not too bad if we consider the approximations used in the theory and the experimental uncertainties of thermal effects and mode frequency.

\section{References}

1. H. Kogelnik, T.Li: Proc. IEEE 54, 1312 (1966)

2. G.J.Ernst, W.J.Witteman: IEEE J. Quantum Electr. QE-9, $911(1973)$

3. L.W.Casperson, A. Yariv: Appl. Phys. Letters 12, 355 (1968)

4. A. G. Fox, T.Li: IEEE J. Quantum Electr. QE-2, 774 (1966)

5. H. Kogelnik: Appl. Optics 4, 1562 (1965)

6. L.W. Casperson: Appl. Optics 12, 2434 (1973)

7. G.J.Ernst, W.J.Witteman: IEEE J. Quantum Electr. QE-10, 37 (1974)

8. A. Y.Cabezas, R. P. Treat: J. Appl. Phys. 37, 3556 (1966) 